\title{
THE PATHOLOGY OF CHOLESTERIN AND FAT DEPOSITION IN MUSTARD GAS INJURIES OF THE CORNEA*
}

\author{
BY
}

\author{
IDA MANN and B. D. PULlinger
}

\begin{abstract}
THE series of changes which precede the onset of delayed mustard gas ulceration of the cornea leads to the deposition of fat and cholesterin in the substantia propria. This degeneration is not specific for dichlorodiethyl sulphide injuries but is well known to occur in man as a sequel to severe keratitis, either infective or due to other chemicals, for example sulphuric acid. Since the process appears to offer an interesting pathological problem apart from its importance in producing secondary breakdown of the scars, it is proposed to consider it in detail from the experimental angle. There are many gaps in our knowledge of the proces's, and at present we are not able to produce it experimentally at will, although it is often possible to predict its appearance during the course of an experiment.
\end{abstract}

- Our observations on its occurrence are based on a series of experiments undertaken to elucidate the problem of delayed mustard gas keratitis, and a detailed account of this has been published (A Study of Mustard Gas Lesions of the Eyes of Rabbits and Men. Ida Mann and B: D. Pullinger, Proc. Roy. Soc., Med. Jan. 1942. Vol. XXXV, No. 3, pp. 229-244.) in the course of this work we produced cholesterin-containing scars in 14 eyes out of a total of 125 possible expèriments. The incidence is not high, and the mechanism of production still somewhat obscure. In the course of our work we studied the effects of the application of varying doses of liquid mustard gas to various anatomical sites, e.g., to the cornea alone, to the limbus and cornea, and to the conjunctiva. The liquid mustard gas was coloured black with Sudan black, and blue-eyed rabbits were used so that the exact sites at which the action occurred could be observed. A full description of the technique is given in the paper referred to above.

In the first place it was noted that central corneal lesions healed without vascularisation of the cornea. Secondly, it was found that such avascular lesions never produced cholesterin or fat in the scars. It is therefore considered that the essential factor in this degeneration is previous vascularisation of the cornea. This vascularisation is of various types, though, in all, the invading vessels are derived from those of the conjunctiva. In some cases,

* Read at the Oxford Ophthalmological Congress, July, 1942. 
merely superficial vascular loops of thin even calibre crossed the limbus. These often extended for a time just beneath the epithelium, and then retrogressed, often disappearing almost completely and never being accompanied by haemorrhage or thrombosis. Such regular superficial vessels were never followed by deposition of fat or cholesterin.

In other cases the vessels" penetrated the substantia propria, which showed separation of the lamellae by oedema fluid ahead of the invading vessels. These deeper vessels showed extremely bizarre shapes, and irregular calibre, and extended in the form of tips or pointed ends, in which no movement of blood could be seen. Bulbous ends and detached tips were formed. Branches and isolated islands of blood appeared and disappeared rapidly, the whole pattern varying from day to day, always extending further into the cornea, and always accompanied by -secondary oedema. The arrangement of bulbous ends and right angled branches suggested the so-called Bowman's canals, formed by

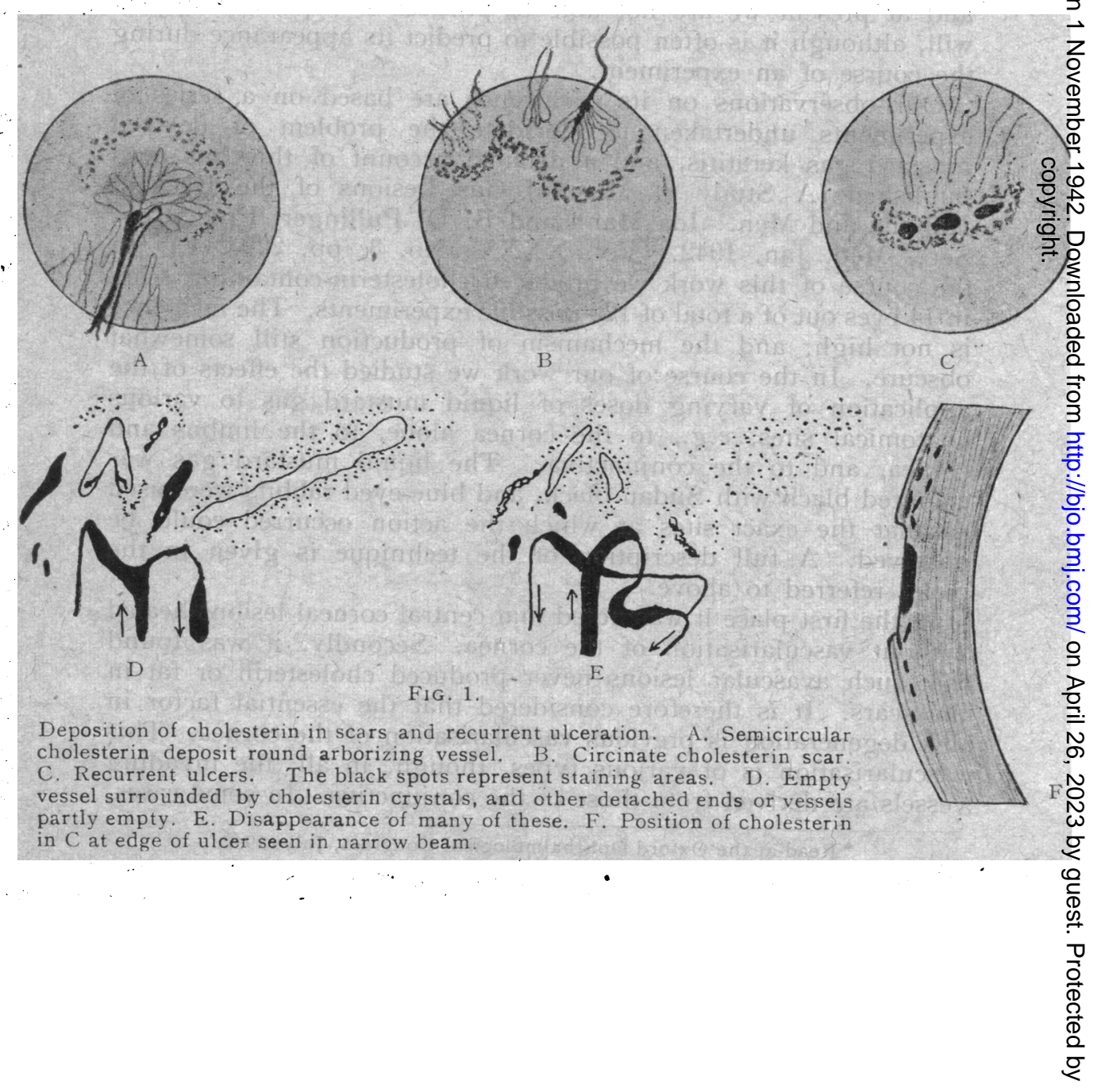


splitting apart of the corneal lamellae by forcing in air. Most of the vessels lay one-third of the way through the cornea. In many cases, detached tips and blood islands became left behind as the condition improved. These sometimes disappeared by haemolysis of the blood within them, which could be observed seeping out through the endothelial wall along the lines of the corneal fibrils. As these vessels retrogressed, in a certain, relatively small number of cases (14 out of 125) a deposit of fat and cholesterin was formed beyond their disappearing tips. Fig. 1* shows this process, and its final stage, secondary loss of epithelium over the most superficial deposits, and casting out of the cholesterin from the floor of the ulcer (delayed keratitis). It will be noted from the figure that various types of deep vessel may be associated with the degenerative process. In $\mathrm{A}$ there is a single large vessel ending in an arborisation around the terminal branchès of which the cholesterin and fat lie in a regular semi-circle. This is not the commonest type of vessel to be associated with the condition. In $\mathrm{B}$ there are three groups of vessels, each surrounded by a circinate deposit, so that a festooned appearance is produced. In $\mathrm{C}$ the vessels are a series of fairly -straight loops. This type is the commonest. As the cholesterin appears, it at first outlines and then obscures the empty endothelial wall of the vessel, which finally disappears completely. The fat precedes the appearance of crystals and becomes rather less in amount as they increase.

In attempting to assess the probabilities of cholesterin formation in any given case, we noticed one peculiar feature, namely, that the series of eyes which received therapeutic treatment (40 out of the 125) showed a much higher percentage of degenerate scars than did those in which the lesion was allowed to run its usual self-limiting course. Forty eyes of the series received varying treatments (irrigations with tap water and saline, drops of-liquid paraffín, glycerine, albucid, bleach solution, sodium bicarbonate, cod liver oil, cod liver oil and sodium bicarbonate, or Bonnefont's solution). Of these 40 eyes 8 developed cholesterin scars, as against 6 in the untreated 85 eyes. In other words, 20 per cent. of the treated eyes showed cholesterin, and 7 per cent. of the untreated. It is therefore possible that continued damage after the initial injury is a factor in the production of these scars.

Unfortunately, in the cases in men gassed in the last war, and now showing delayed keratitis, it is not possible to discover in detail what treatment was used. The present appearances in such cases are very similar to those in rabbits, except that the deposits are much more irregular, the semicircular line around a single arborising vessel not having been noted. This would be expected,

\footnotetext{
${ }^{*}$ Fig. 9 from the Proc. Roy. Soc. Med. paper.
} 
since the experimental application of the gas is more localised than the accidental.

The identification of cholesterin was made in the first place by the slit-lamp, and the presence of cholesterin and fat was subsequently confirmed microscopically. With increased.experience of fatty scars it became possible to distinguish them also and to make a differential diagnosis between fatty and cellular scars.

\section{The Distribution and Nature of the Lipoid Deposits}

The' lipoid occurs in two forms :-

1. As exceedingly fine droplets like an emulsion bathing the fibrils of the substantia propria. The fibrils themselves appear to remain intact. More work using silver impregnation requires to be done to settle this point. The droplets are stained with the usual fat.stains, e.g., Scharlach R, Sudan III and IV and Sudan black. They are not doubly refracting in polarised light. They occur at any level of the substantia propria.

This distribution of lipoid was found in a sulphuric acid burn in a human eye which we had the opportunity of examining.

2. As large intracellular globules and crystals having the characters of esters of cholesterin. These are :-

(a) Double refraction in polarised light.

(b) Appearance of double "Maltese crosses" in polarised light.

(c) Disappearance of double refraction on heating.

(d) Reappearance of double refraction and of crystals on cooling.

(e) Capacity to be stained with the usual fat stains.

The cells containing the crystalline lipoid are macrophages. They appear in and around the deposits of emulsified fat, also when no emulsion is present.

3. As fine intracellular globules of isotropic fat in adventitial cells and possibly endothelial cells also of blood capillaries in eyes that have, and in others that have no actual "lipoid scars."

4. As free cholesterin crystals with :-

(a) A characteristic appearance in the slit-damp beam.

(b) Double refraction in polarised light.

(c) Crystalline structure when seen with high microscopic magnifications, viz. flat crystals with corners " bitten out."

The emulsified lipoid and the crystalline lipoid may be quite distinct or may be stages of a process related in time. Appearances suggest that the fine globules are ingested by macrophages and converted by them into cholesterin esters. Further work is required to settle this point.

Another problem of general pathology on which the history of these lipoid scars throws some light is that of atheroma. The intima of blood vessels in which intra- and extra-cellular lipoid deposits occur, is another non-vascular or relatively 'non-vascular 
anatomical structure. Just how far it is or is not vascular in normal man is not completely clear. In certain animals it is a-vascular and in man this is probably so. The abundant vasa vasorum supply the adventitia rather than the intima.

In the cornea, lipoid deposition is the last event in a series of changes following chemical damage of various kinds. Let the chemical be called $X$. The chemical $X$ does not itself cause these deposits for they never appear unless the cornea vascularises, and then only occasionally. The vessels or something which accompanies them are responsible for the lipoid accumulation. Invasion of the cornea by wandering cells alone does not account for the lipoid scars because all central non-vascular lesions heal with the aid of wandering cells but deposition of lipoid is never a feature of these scars. Thus the sequence of events is clear up to a point in the cornea.

In atheroma the sequence of events is not yet known. It is often assumed or suggested on the basis of animal experiments that the intimal lipoid deposits are directly due to hypercholesterinaemia. Our observations on the cornea indicate that a hypothetical substance or substances $\mathrm{X}$, may, by reaching the intima in the first place (or, if a vitamin, by failure to reach it) cause sufficient damage to bring in capillaries as part of an inflammatory reaction. This vascularisation, which is often seen in atheroma, may be followed by regression and by deposition of lipoid. This is merely speculation but, granted that the intima is normally non-vascular, it does not disagree with the facts.

\title{
ANOMALOUS PUPIL REACTIONS
}

BY

\author{
B. G. Edelston, M.D.ED., B.CH. \\ TUBERCULOSIS OFFICER, KENT COUNTY COUNCIL
}

ONE of the most instinctive, protective actions of human behaviour is the raising of the hands to the eyes to guard them from an excessive sudden access of light; and this action is in health accompanied by the reflex shutting down of the aperture of the pupil in a miosis that is both protective and utilitarian. The pupil of Argyll Robertson is a strange paradox : but a stranger one still is the actual dilatation of the pupils to the stimulus of light which occurs, very rarely, in tabetic states. The three cases described below were seen in the course of the work at Chest Clinics over a period of ten years, and it is thought that their rarity and the obscure pathological mechanism of their causation may be found of clinical and speculative interest. 